\title{
Antenna Arrays Principle and Solutions: Robust Control Approach
}

\author{
Florin Hutu, Sebastien Cauet, Patrick Coirault
}

\begin{abstract}
This paper treats solutions on the ability of a chain of non identical oscillators to drive antenna arrays. Frequency approaches were studied in order to solve the problem of synchronization of the oscillators. However, in this article, a new structure of chain of oscillators is introduced. Secondly, Lyapunov theory of stability is used to design a dynamical controller guarantying the oscillators synchronization. The problem of synchronization is transformed into a problem of asymptotic stabilization for a nonlinear system. It is formulated as a system of linear matrix inequalities where the parameter variations of the two oscillators and their differences are modeled by polytopic matrices. The theoretical result is successfully applied to an array of transistor-based oscillators used in "smart antenna" systems.
\end{abstract}

Keyword : Nonlinear systems, Control applications, Antenna arrays

\section{Introduction}

The demand of mobile communication services is in a continuous growth, moreover, it is estimated that the rate will be maintained in the next years. This continuous development has stimulated the research of new hardware and software solutions in order to increase the volume of exchanged data and a better management of the emitted or received electromagnetic field.

Smart antenna arrays comprise a number of antennas that work in conjunction with an intelligent system that processes the received and transmitted data. The processing can be realized in a hardware or in a software way and allows smart antenna arrays to focus beams into particular directions.

This problem can be partially solved by using several directional antennas. This solution divides the 360-degree coverage area into sectors. However, smart antenna arrays provide a much more effective solution by focusing the transmitted power toward user and only looking in the direction of the user for the up link signal. This ensures that the user receives the optimum quality of service and the maximum coverage from a base station.

The new technologies development increases the antenna array performances and minimizes the costs of production and the occupied space. It makes them implementable in domains like wireless or satellite communications, radar systems, missile defense systems, automobile industry, etc.

Smart antennas or antenna arrays are a part of communication systems that can improve their global performances. This technique can increase the spectral efficiency and reduce the multi path fading, bit error rate (BER), the co-channel interferences (CCI) and the system complexity [1]. This is possible by electronically adjusting the beam pattern of the antenna array in order to provide important gain for the desired signals and small gain for interference signals.

At emission, the purpose of smart antennas is to minimize the interference between the different transmitters who works on the same communication channel and, thus to more efficiently use the emitted power. For this reason, the beam shape must be controlled in order to minimize the amplitude of the side lobes and to maximize the energy in the main lobe. Moreover, the direction of the main lobe must be controlled. the focused application is inter-vehicle communication. The bandwidth will be, first of all, in the area of $24 \mathrm{Ghz}$ and finally around $79 \mathrm{GHz}$. At these frequencies, technique like "software defined radio" can not be used. One of the main objectives that is pointed out is to develop a structure which will extend in both space and time the safety information available to drivers by using the infrastructure and vehicles as sources. 
When the smart antennas are used in reception systems, the signals coming from interference directions must be rejected and those which comes from the desired directions must be privileged. For this reason, different phases and amplitudes must be assured by the carrier signals locally generated [2, 3, 4].

The work that has been done in the field of dynamics of coupled nonlinear systems using the frequency approach [5][6,7][8] shows that they offer methods of phase control among array elements and beam scanning capabilities but also implies problems of stabilization.

The proposed structure of the array of antennas is based on unidirectional coupled oscillators.

In details, this paper treats the synchronization of a system made by two oscillators with an unidirectional coupling and this problem of synchronization is transformed in a problem of stabilization for a nonlinear system. The strategy chosen is to find an output feedback dynamic controller using Lyapunov functions that assures a robust synchronization despite parameters variations of the oscillators.

The problem of computing dynamic output feedbacks on LTI (linear time-invariant) systems in term of matrix inequalities is difficult to solve. There are two known techniques: the iterative algorithms and the cancellation of variable products by using the matrix separation lemma. The reader can found some papers on treatment of this problem by LMI (Linear Matrix Inequality)-s who can be numerically solved $[9,10,11,12,13]$.

The variations of the parameters of the oscillators are taken into account by considering the state matrix as a polytopic one. The polytopic structure is easily tractable by Linear Matrix Inequalities. Once the stability of the vertices, defined for the polytope is proved, the stability and the synchronization of the two oscillators is assured for all systems inside the polytope.

The nonlinear character of the oscillators allows the synchronization (if their free running frequencies are in a certain domain [6]) but also makes them dependent of initial conditions. The main objective is to cancel the nonlinear effect and to maintain the synchronization when the physical parameters of the oscillators and external conditions are modified. The originality of the method comes from the inclusion of the non-linear term and the undesired variations in a perturbation. This problem is transformed in a $\mathcal{H}_{\infty}$ optimization.

In section 2 is presented an overview of the antenna array theory. In section 3 a model of Master and Slave oscillators and the controller synthesis are introduced. Section 4 presents the numerical results in the case of an array of coupled nonlinear oscillators.

\section{Antenna array theory overview}

\subsection{Theoretical background}

A smart antenna is composed by an array of individual radiative elements (elementary antennas), which are placed in a particular configuration (linear, circular or matrix). By gathering these elementary antennas in such arrays and by changing the characteristics of the signals associated to each element, the array can present different gains according to the direction.

Let us consider an uniform linear array of $N$ identical patch antennas placed at the same distance $d$ between them as in Fig. 1.

For the theoretical study of this configuration, it is assumed that in the elementary antennas, harmonic signals of the same frequency but different amplitudes and phases are injected.

The mathematical expression of the total electromagnetic field generated by the array in far-field regions (Fraunhofer regions) can be written as the product of the electromagnetic field of the reference antenna (which is considered the first antenna in the array) and a term which depends on the amplitudes and phases of the injected signals

$$
\mathbb{E}_{\text {total }}=\mathbb{E}_{\text {ref }} * f(\Theta) .
$$

Thereafter, the gain of the antenna array will be considered, knowing that it is a normalization of the 


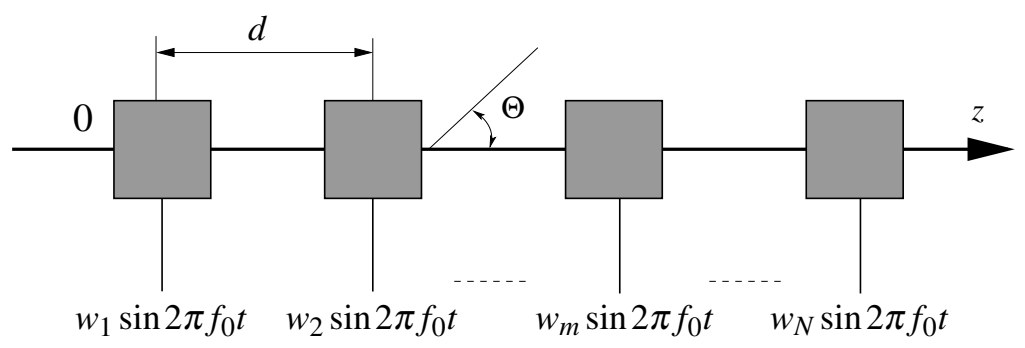

Figure 1: An uniform antenna array

amplitude of the electromagnetic field. The array factor can be written as

$$
f(\Theta)=\sum_{m=1}^{N} \mathbf{w}_{\mathbf{m}} e^{-j(m-1) k_{0} d \cos \Theta},
$$

where $\mathbf{w}_{\mathbf{m}}=A_{m} e^{j \varphi_{m}}$.

In the easiest emission case, the amplitudes have the same value, the mathematical expression of the radiation pattern is

$$
f(\theta)=\frac{1}{N} \frac{\sin \frac{N \gamma}{2}}{\sin \frac{\gamma}{2}},
$$

where $\gamma=\varphi-k_{0} d \cos (\theta), N$ the number of antennas, $d$ the distance between them. It can be seen that a quantity of the radiated energy is lost in the side lobes which implies a certain weakening of the antenna array gain.

It is obvious that in the emission case, both variations of the amplitudes and the phases of the carrier signals are in a large interval. This paper proposes a new technique to generate such signals, which have the same frequency and different phases and amplitudes. It can be concluded that both variations of the amplitudes and phases are in a large interval.

\section{Problem formulation}

\subsection{Problem statement}

If the amplitude variation can be easily solved by using variable gain amplifiers, the problem of the phase variation is more constraining.

There are several techniques which permits solving this problem. The main technical problem with beamforming for transmit is realizing the phase and amplitude of the signals in each antenna channel. The first step will be generating the reference signal with the selected or required frequency. It has to be modulated with the information to be transmitted (e.g. radar, communication). Then it has to be amplified and distributed to all transmit channels. Then the individual signals have to be weighted for beamforming, that means amplified or attenuated, according to the desired weighting amplitude. The desired phase has to be realized by a suitable steerable phase shifter or delay line. Digital devices are now available, under the headline "software defined radio". This technique is not usable on frequencies over $400 \mathrm{Mhz}$. Here, techniques, which are pointed out, are designed for applications over $2 \mathrm{GHz}$.

Techniques can be divided into both main approaches. The first approach uses the signal generated by one oscillator and the second which uses signals generated by array of coupled oscillators.

Signals with the same frequency but different phases and amplitudes can be built by delaying the signal generated with one master oscillator using high-frequency power dividers and variable delay lines or Butler couplers. This approach is very useful when discrete-time systems are built. Another approach 
is to use polyphasic oscillators and a multiplexing system [5]. But in these techniques, it can not be obtained continuous phase variations.

The second approach is based on the synchronization of arrays of oscillators having their free running frequencies with a weak dispersion. In $[6,8]$ it was demonstrated that arrays of coupled nonlinear oscillators can synchronize. Moreover, according to the coupling strength and to free-running frequencies, phase variations can be made. Recent works [14] shows how the phase variation can be guaranty by changing only the free-running frequencies of all coupled oscillators in the array.

In order to generate these carrier signals, the following general schematic Fig. 2 is proposed.

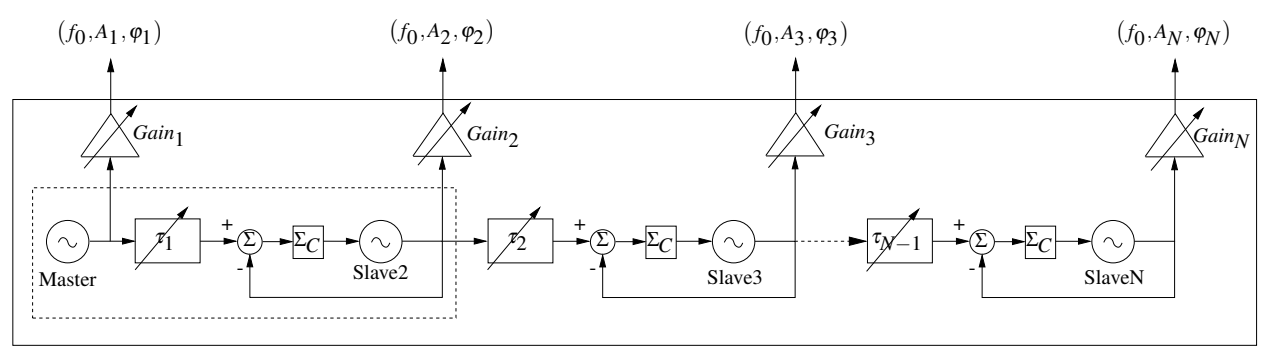

Figure 2: Unidirectional coupling of a chain of oscillators

This schematic is a variation of the York's approach shown in [15]. Because of the unidirectional coupling, each slave oscillator is driven only by its left neighbor. Hence, the study of this configuration is reduced to the study of a pair of two non-linear systems (Master-Salve synchronization). The purpose is to design the parameters of the controller system $\Sigma_{C}$ in order to make the output $y_{e}(t)$ tends toward zero. When this objective is fulfilled, the delayed output of the master oscillator and the output of the slave oscillator become identical, so both oscillators are synchronized.

Generally, because of the technological realization, the oscillators don't have the same free running frequencies. This is the reason why the feedback loop was introduced to guarantee the robust synchronization between both oscillators. The delay element and the variable gain amplifier will guaranty different phases and amplitudes for the output signals.

The difference between the oscillators will be modeled as a variation of the slave oscillator parameters around those of the master oscillator parameters, which is considered as the reference. The variations due to the temperature or at the ageing of the components are modeled by a polytopic uncertainty of the master oscillator parameters around the nominal values.

The oscillators are built using a double differential pair structure. In order to determine the parameters of the dynamical controller, the nonlinear oscillators is modeled using the van der Pol model.

In this article, we consider that the system is a perturbed van der pol model as:

$$
\left\{\begin{array}{l}
\dot{x}=A\left(\theta_{1}\right) x+g\left(x, t, \theta_{1}\right)+B u \\
y=C x
\end{array}\right.
$$

where

$$
\begin{array}{ccc}
x=\left[\begin{array}{c}
i_{L} \\
v_{0}
\end{array}\right] & A\left(\theta_{1}\right)=\left[\begin{array}{cc}
0 & \frac{1}{L_{0}} \\
-\frac{1}{C_{0}} & 0
\end{array}\right] \quad \begin{array}{c}
u=\frac{i_{\text {inj }}}{C_{0}} \\
g\left(x, t, \theta_{1}\right)=\left[\begin{array}{c}
0 \\
\frac{\alpha}{C_{0}} x_{2}-\frac{\beta}{C_{0}} x_{2}{ }^{3}
\end{array}\right]
\end{array} \quad B=\left[\begin{array}{c}
0 \\
1
\end{array}\right] \quad C=\left[\begin{array}{ll}
0 & 1
\end{array}\right],
\end{array}
$$

with the uncertain parameters $\theta_{1}=\left[\alpha, L_{0}, C_{0}\right]$. 


\subsection{Master-slave synchronization}

The structure is made up by two different systems which belong to the class previously described. The master system is considered independent $(u=0)$ and the dynamical controller $\Sigma_{C}$ drives the slave system using the error signal as reference. The error signal is constituted by the difference between a delayed version of the master output and the slave output.

The state-space representation of the master system can be written as follows

$$
\Sigma_{M}:\left\{\begin{array}{l}
\dot{x_{M}}=A_{M}\left(\theta_{1}\right) x_{M}+g_{M}\left(x_{M}, t, \theta_{1}\right) \\
y_{M}=C x_{M}
\end{array} .\right.
$$

For the slave system, the state-space representation can be written as

$$
\Sigma_{S}:\left\{\begin{array}{l}
\dot{x_{S}}=A_{S}\left(\theta_{1}\right) x_{S}+g_{S}\left(x_{S}, t, \theta_{1}\right)+B_{2} u \\
y_{S}=C x_{S}
\end{array} .\right.
$$

Between the parameters of the master and the slave oscillators, it is considered that there is the same difference $\delta$

$$
\left\{\begin{array}{l}
L_{S}=L_{M}(1+\delta) \\
C_{S}=C_{M}(1+\delta) \\
\alpha_{S}=\alpha_{M}(1+\delta) \\
\beta_{S}=\beta_{M}(1+\delta)
\end{array} .\right.
$$

In that case, this notation can be introduced

$$
A_{M}\left(\theta_{1}\right)=A_{S}\left(\theta_{1}\right)+B_{1}\left(\theta_{2}\right) .
$$

This difference is transformed into the difference between the state matrix of the master and the slave. With the assumptions in $(8), B_{1}\left(\theta_{2}\right)$ can be written as follows

$$
B_{1}\left(\theta_{2}\right)=\left[\begin{array}{cc}
0 & -\frac{\delta}{L_{M}(1+\delta)} \\
\frac{\delta}{C_{M}(1+\delta)} & 0
\end{array}\right] .
$$

If an error state is defined as

$$
e(t)=x_{M}(t-\tau)-x_{S}(t),
$$

a state-space representation can be written

$$
\Sigma_{e}:\left\{\begin{array}{l}
\dot{e}=A_{M}\left(\theta_{1}\right) e-B_{1}\left(\theta_{2}\right) x_{s}+e_{g}\left(x_{M}, x_{S}, t, \theta_{1}\right)-B_{2} u \\
y_{e}=C e
\end{array},\right.
$$

where

$$
e_{g}\left(x_{M}, x_{S}, t, \theta_{1}\right)=g_{M}\left(x_{M}, t, \theta_{1}\right)-g_{S}\left(x_{S}, t, \theta_{1}\right) .
$$

\subsection{Nonlinear bound determination}

In order to determine the bounds of the nonlinearities difference, the scalar function $f: \mathcal{D}_{1} \mapsto \mathcal{D}_{2}$ $f(x)=-\alpha x+\beta x^{3}$ is used. The bounds can be considered as the slopes of the tangents passing through $x=x_{m}$ and $x=0$ of $f(x)$.

$$
\begin{array}{r}
-\alpha\left(x_{2}-x_{1}\right) \leq\left(f\left(x_{2}\right)-f\left(x_{1}\right)\right) \leq\left(-\alpha+3 \beta x_{m}{ }^{2}\right)\left(x_{2}-x_{1}\right) \\
\forall x_{1}, x_{2} \in \mathcal{D}_{1}
\end{array}
$$


Consider both nonlinear oscillators and the domain $\mathcal{D}_{1}=[-1.35 \mathrm{~V}, 1.35 \mathrm{~V}]$, then the bound of the nonlinearities difference (13) can be written as follows

$$
\left[\begin{array}{c}
0 \\
-\frac{1}{C_{0}}\left(\alpha+3 \beta-0.2^{2}\right)
\end{array}\right] \leq e_{g}\left(x_{M}, x_{S}, t, \theta\right) \leq\left[\begin{array}{c}
0 \\
\frac{\alpha}{C_{0}}
\end{array}\right] .
$$

\subsection{Controller synthesis}

Assume that $A_{M}\left(\theta_{1}\right)$ resp. $B_{1}\left(\theta_{2}\right)$ are two matrices that belong to a polytope of matrices and it is represented by a convex combination of the extreme matrices $A_{i}$ resp. $B_{1 i}$ with $i=1 \ldots 2^{M}$.

$$
\mathcal{A}_{M}=\left\{A_{M}\left(\theta_{1}\right) \mid A_{M}\left(\theta_{1}\right)=\sum_{i=1}^{2^{M}} \xi_{i} A_{i} ; \xi_{i} \in \Delta_{1}\right\}
$$

and consider that the matrix $e_{g}\left(x_{M}, x_{S}, t, \theta_{1}\right)$ can be bounded with $N_{B}\left(\theta_{1}\right)$ being its upper bound

$$
e_{g}\left(x_{M}, x_{S}, t, \theta_{1}\right) \leq N_{B}\left(\theta_{1}\right) e .
$$

The worst case for our system is the superior limit, then the matrix $A_{N}(\theta)=A_{M}\left(\theta_{1}\right)+N_{B}\left(\theta_{1}\right)$.

Assume that the dynamical output controller of the system (12) is described by the following statespace representation and its dimension is $n_{c}$.

$$
\Sigma_{C}:\left\{\begin{array}{l}
\dot{x_{c}}=A_{c} x_{c}+B_{c} y_{e} \\
u=C_{c} x_{c}+D_{c} y_{e}
\end{array}\right.
$$

The purpose of this controller is to make the slave system follow the delayed output of the master system. This condition is performed when the error signal defined in (11) tends toward zero. The term $B_{1}\left(\theta_{2}\right) x_{s}$, representing the difference between both systems, acts as a perturbation on the error state $e$.

The synthesis of this controller has been made with a technique similar to $[9,10]$ The following theorem solves the problem of variable matrices product in the synthesis problem by introducing extra unknown variable matrices.

Theorem 1. If there exists a set of matrices $P_{i}>0$, a state feedback controller $K_{0}$, an unknown variable square and nonsingular matrix $G \in \mathbb{R}^{n_{u}+n_{c}}$, an unknown variable matrix $H \in \mathbb{R}^{\left(n_{u}+n_{c}\right) \times\left(n_{u}+n_{c}\right)}$ and four unknown variables matrices $F_{1}, F_{4} \in \mathbb{R}^{\left(n_{x}+n_{c}\right) \times\left(n_{x}+n_{c}\right)}, F_{2} \in \mathbb{R}^{n_{x} \times\left(n_{x}+n_{c}\right)}$ and $F_{3} \in \mathbb{R}^{\left(n_{\infty}+n_{c}\right) \times\left(n_{x}+n_{c}\right)}$ such that the inequality (19) is verified, then the dynamical controller $K=G^{-1} L$ makes the error system (12) asymptotically stable for all matrices $A_{N}\left(\theta_{1}\right)$ and $B_{1}\left(\theta_{2}\right)$ described as a polytope.

$$
\begin{gathered}
\Phi_{2}+{ }^{1} \operatorname{Sym}\left\{\left[\begin{array}{l}
F_{1} \\
F_{2} \\
F_{3} \\
F_{4} \\
\mathbb{O}
\end{array}\right]\left[\begin{array}{lllll}
\mathbb{O} & \mathbb{O} & \mathbb{O} & \mathbb{O} & \widetilde{B}_{2}
\end{array}\right]\right\}+\operatorname{Sym}\left\{\left[\begin{array}{l}
\mathbb{O} \\
\mathbb{O} \\
\mathbb{O} \\
\mathbb{O} \\
\mathbb{I}
\end{array}\right] L\left[\begin{array}{lllll}
\widetilde{C} & \mathbb{O} & \mathbb{O} & \mathbb{O} & \mathbb{O}
\end{array}\right]\right\} \\
+\operatorname{Sym}\left\{\left[\begin{array}{l}
\mathbb{0} \\
\mathbb{O} \\
\mathbb{O} \\
\mathbb{O} \\
\mathbb{I}
\end{array}\right] G\left[\begin{array}{lllll}
-K_{0} & \mathbb{O} & \mathbb{O} & \mathbb{O} & -\mathbb{I}
\end{array}\right]\right\}<\mathbb{O}
\end{gathered}
$$


The matrix $\Phi_{2}$ is defined as follows

$$
\Phi_{2}=\left[\begin{array}{ccccc}
\mathbb{O} & \mathbb{O} & C_{c l}{ }^{T} & P_{i} & \mathbb{O} \\
\mathbb{O} & -\gamma \mathbb{I} & \mathbb{O} & \mathbb{O} & \mathbb{O} \\
C_{c l} & \mathbb{O} & -\gamma \mathbb{I} & \mathbb{O} & \mathbb{O} \\
P_{i} & \mathbb{O} & \mathbb{O} & \mathbb{O} & \mathbb{O} \\
\mathbb{O} & \mathbb{O} & \mathbb{O} & \mathbb{O} & \mathbb{O}
\end{array}\right]+\operatorname{Sym}\left\{\begin{array}{c}
\left.\left[\begin{array}{c}
F_{1} \\
F_{2} \\
F_{3} \\
F_{4} \\
\mathbb{O}
\end{array}\right]\left[\begin{array}{ccccc}
\widetilde{A}_{0 i} & \widetilde{B}_{1 j} & \mathbb{O} & -\mathbb{I} & \mathbb{O}
\end{array}\right]\right\}, \\
\forall i \in\left\{1 \ldots 2^{M}\right\} \text { and } \forall j \in\left\{1 \ldots 2^{P}\right\}
\end{array}\right.
$$

where $\widetilde{A}_{0 i}=\widetilde{A}_{N i}+\widetilde{B} K_{0}$ and

$$
K=\left[\begin{array}{l|l}
D_{c} & C_{c} \\
\hline B_{c} & A_{c}
\end{array}\right]
$$

The expression (16) can be numerically solved using Matlab's ${ }^{\circledR}$ "LMI Toolbox".

\section{Numerical results}

In order to check the theoretical result, a transistor-based simulation has been done using Agilent's $\mathrm{ADS}^{\odot}$ software and MOSFET transistors in $0.35 \mu \mathrm{m}$ silicon technology.

It was considered that all the parameters of $A_{N}(\theta)$ have $\pm 5 \%$ variation around their nominal values. This variation can be seen as the variation depending on the temperature of the oscillators that are built on the same integrated circuit substrate. This is mathematically transformed into the variation of the state matrix $A_{N}\left(\theta_{1}\right)$ inside the polytope. Using the Matlab's "LMI Toolbox" applied to the 8 vertices of the polytope, the following output-feedback controller was found

$$
K=\left[\begin{array}{l|l}
D_{c} & C_{c} \\
\hline B_{c} & A_{c}
\end{array}\right]=\left[\begin{array}{c|c}
2.66588 \cdot 10^{11} & 22.3994 \\
\hline 7.51499 \cdot 10^{9} & -1.1885
\end{array}\right] .
$$

It assures the synchronization of oscillators having $\delta= \pm 5 \%$ difference between parameters. This difference is represented by variation of the perturbation matrix $B_{1}\left(\theta_{2}\right)$ inside the polytope.

Variations between $L_{M}, C_{M}$ and $L_{S}, C_{S}$ parameters, corresponds to a possible difference between the free-running frequencies of both oscillators

$$
f_{0 S} \in\left[f_{0 M}(1-|\delta|)^{2} f_{0 M}(1+|\delta|)^{2}\right] .
$$

The difference between $\alpha_{M}, \beta_{M}$ and $\alpha_{S}, \beta_{S}$ stands for a possible difference between the transistor operating points of both nonlinear oscillators.

This controller was applied to a pair of both non-linear oscillators. Their free running frequencies are $f_{0 M}=2 \mathrm{GHz}$ and $f_{0 S}=2.2 \mathrm{GHz}$. It has been chosen those frequencies in order to build a discrete component platform.

In fig. 4 are presented both output voltages for master and slave oscillators and is divided into three sequences. a first sequence in which, the controller $\Sigma_{c}$ is not activated, both oscillators oscillates from their free-running frequencies. the second step at $t=55 \mathrm{~ns}$, the controller $\Sigma_{c}$ is activated. The obtained delay is closed to the imposed value $\left(\tau=T / 4=1.25 \cdot 10^{-10} s\right)$. This delay will correspond to a orientation of the main lobe in $\Theta=120^{\circ}$. Finally, at $t=60 \mathrm{~ns}$, in order to verify the robustness of the dynamical controller, the free running frequency of the master oscillator was changed to $f_{M}=2 \mathrm{GHz}$.

In fig. 3 the error between both output signals is presented. It can be seen that the error tends toward zero after a short period of time when the controller is started.

\footnotetext{
${ }^{1} \operatorname{Sym}\{X\}=X^{T}+X ; \forall X \in \mathbb{R}^{n}$
} 


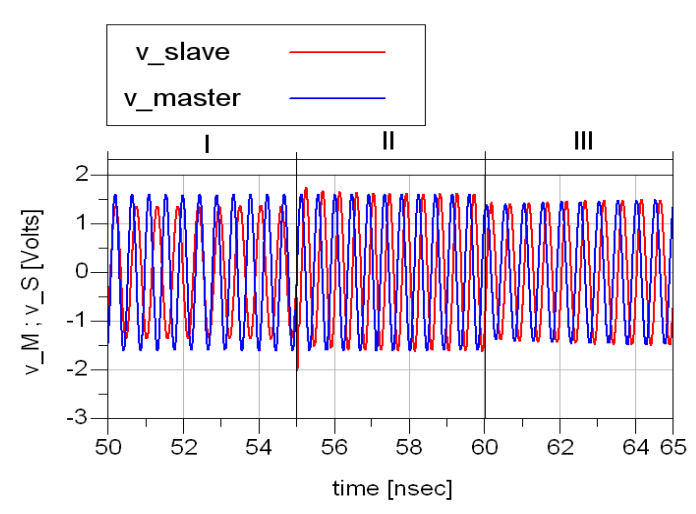

Figure 3: Output voltages of both oscillators

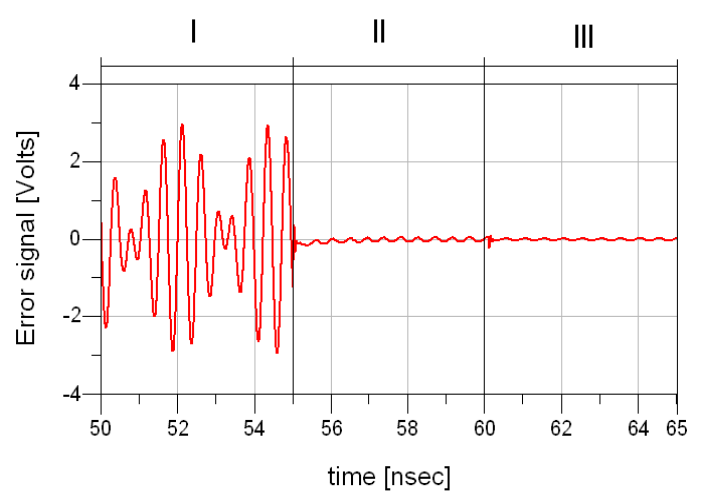

Figure 4: The error between both signals provided by the oscillators

\subsection{Array of oscillators}

Consider the situation where the $\Theta_{p}=60^{\circ}$ direction must be privileged and $\Theta_{i 1}=90^{\circ}$ and $\Theta_{i 2}=120^{\circ}$ must be rejected.

In table 4.1, the necessary and final values of the amplitudes and phases are shown for $N=8$ antennas. Figure fig.5 depicts the corresponding radiation pattern. The orientation of the main lobe is closed to the desired value $\Theta_{p}=58.14$ and both interference directions $\Theta_{i 1}=90^{\circ}$ and $\Theta_{i 2}=120^{\circ}$ are rejected.

\section{Conclusion}

This paper presents a novel method to drive antenna arrays. It is based on unidirectionally coupled oscillators. An output feedback controller has been designed to assure synchronization with advanced control theory using LMI (Linear Matrix Inequalities) tools. The result was successfully extended to a chain of eight unidirectionally coupled oscillators. Additional research will be made to constrain the dynamical controller to realize the desired delay in order to eliminate the delay element. 


\begin{tabular}{|c|c||c|c|}
\hline \multicolumn{2}{|c||}{ Amplitudes $[V]$} & \multicolumn{2}{c|}{ Phases $\left[^{\circ}\right]$} \\
\hline necessary & simulated & necessary & simulated \\
\hline 0.269 & 0.21 & 0 & 0 \\
0.21 & 0.2 & 143 & 138 \\
0.14 & 0.14 & 180 & 179 \\
0.4 & 0.4 & -162 & 160 \\
0.08 & 0.08 & 0 & -2 \\
0.14 & 0.13 & 63.1 & 57 \\
0 & 0 & 0 & 7 \\
0.3 & 0.29 & -24.4 & -30 \\
\hline
\end{tabular}

Table 1: computed and final values for the amplitudes and phases

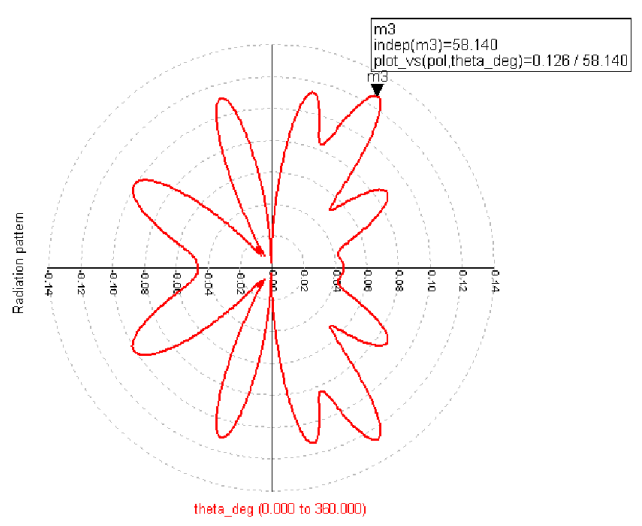

Figure 5: The radiation pattern in the particular case of $\Theta_{p}=60^{\circ}$ 


\section{Bibliography}

[1] L. C. Godara (Ed.), Applications of Antenna Arrays to Mobile Communications, Part I: Performance Improvement, Feasability and System Consideration, Vol. 85, Proceedings of the IEEE, 1997.

[2] S. Chandran, Adaptative antenna arrays Trends and applications, Springer-Verlag, 2004.

[3] S. J. Orfanidis, Electromagnetic waves and antennas (2004). http://www.ece.rutgers.edu/ orfanidi/ewa/

[4] R. C. Hansen, Phased Array Antennas, Wiley-Interscience, 2001.

[5] X. Guan, H. Hashemi, A. Hajimiri, A fully integrated $24 \mathrm{GHz}$ eight-element phased-array receiver in silicon, IEEE Journal of Solid-State Circuits 39 (12) (2004) 2311-2320.

[6] P. Liao, R. A. York, A six-element beam scanning array, IEEE Microwave and Guided Wave Letters 4 (1) (1994) 20-22.

[7] A. Tombak, A. Mortazawi, A novel low-cost beam-steering technique based on the extended resonance power dividing method, IEEE Transactions on Microwave Theory and Techniques (2003) $1-7$.

[8] R. A. York, T. Itoh, Injection and phase-locking techniques for beam control, IEEE Transactions on Microwave Theory and Techniques 46 (11) (1998) 1920-1929.

[9] D. Arzellier, D. Peaucelle, S. Salhi, Robust static output feedback stabilization for polytopic uncertain systems: improving the guaranteed performance bound, in: ROCOND Milan-Italy, 2003.

[10] D. Mehdi, E. Boukas, O. Bachelier, Static output feedback design for uncertain linear discrete time system, IMA Journal of Mathematical Control and Information.

[11] T. Iwasaki, R. E. Skelton, K. Grigoriadis., A unified algebraic approach to linear control design, Taylor and Francis, 1998.

[12] D. Peaucelle, D. Azellier, An efficient numerical solution for $\mathcal{H}_{2}$ static output feedback synthesis, Europeen Control Conference.

[13] S. Boyd, L. E. Ghaoui, E. Feron, V. Balakrishnan, Linear Matrix Inequalities in System and Control Theory, Vol. 15, Studies in Applied Mathematics, USA, 1994.

[14] T. Heath, Simultaneous beam steering and null formation with coupled, nonlinear oscillator arrays, IEEE Transactions on Antennas and Propagation 53 (6) (2005) 2031-2035.

[15] R. A. York, Nonlinear analysis of phase relationshiops in quasi-optical oscillator arrays, IEEE Transactions on Microwave Theory and Techniques 41 (10) (1993) 1799-1809.

Florin Hutu

University of Poitiers

Department : LAII-ESIP

40 avenue du Recteur Pineau

E-mail: florin.hutu@etu.univ-poitiers.fr

Received: November 28, 2007 

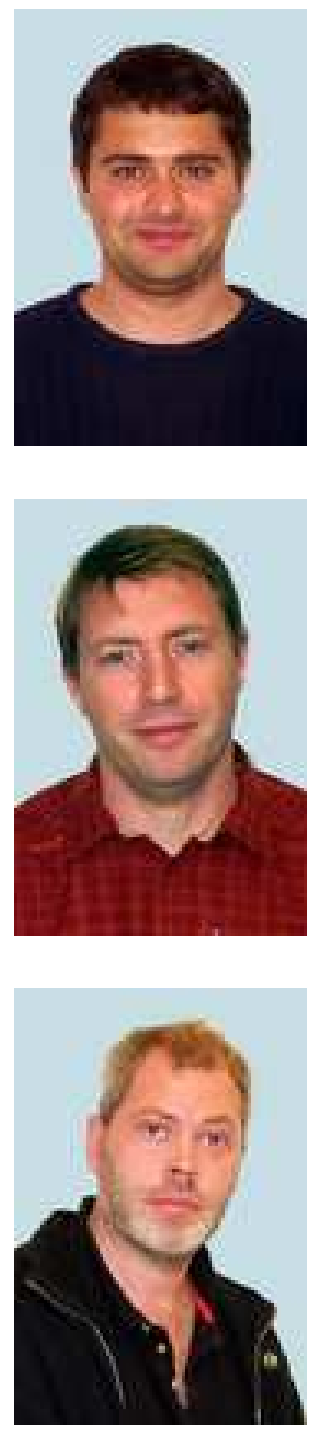

Florin Hutu was born in Romania in 1977 and received the Ph.D. degree in Automatic control in 2007. He is currently a Post doc at the University of Poitiers where his interests are currently involved with the design of microwave antenna array.

Sébastien Cauet was born in France in 1971 and received the Ph.D. degree in Automatic control in 1999. He is currently an associated professor of electrical and computer engineering at the University of Poitiers where his interests are currently involved with the control of electrical power rotating motors, the design of microwave antenna array and application of control to chaos transmission.

Patrick Coirault was born in Poitiers, France, in 1965. He received the Ph.D. degree from the University of Poitiers in 1991. He was an Assistant Professor at the Institute of Technology of Poitiers from 1992 to 1997. He is now Professor at the Institute of Technology of Chatellerault. His current Research interests are in nonlinear identification and control, with applications to chaotic systems. 